\title{
Agôn
}

Revue des arts de la scène

$6 \mid 2013$

La Reprise

\section{Nijinski chorégraphe}

Quelles mémoires et quelles histoires pour quels enjeux?

\section{Roland Huesca}

\section{(2) OpenEdition}

Journals

Édition électronique

URL : http://journals.openedition.org/agon/2730

DOI : $10.4000 /$ agon.2730

ISSN : 1961-8581

Éditeur

Association Agôn

Référence électronique

Roland Huesca, « Nijinski chorégraphe », Agôn [En ligne], 6 | 2013, mis en ligne le 14 février 2014, consulté le 06 mai 2019. URL : http://journals.openedition.org/agon/2730 ; DOI : 10.4000/agon.2730

Ce document a été généré automatiquement le 6 mai 2019.

Association Agôn et les auteurs des articles 


\title{
Nijinski chorégraphe
}

\author{
Quelles mémoires et quelles histoires pour quels enjeux?
}

\author{
Roland Huesca
}

Désirs de mémoire, de reconstitution ou encore de commémoration, depuis la fin du XX siècle, la société française s'enivre de son passé. L'« année du patrimoine » décidée par Giscard d'Estaing alors président de la République en 1980, symbolise à sa manière ce mouvement. Préserver, montrer, réhabiliter, reconstruire, remonter, etc., deviennent les gestes et les mots d'un récit politique et social qui, au présent, interroge le passé. Émergent alors de nouvelles réalités mues par des prises de position et des savoirs singuliers. Substrat de la mémoire collective, ces usages de l'art témoignent à leurs manières de la façon dont une société consigne ses souvenirs, et d'un même élan, institue sa permanence.

2 En une série d'ouvrages désormais célèbres ${ }^{1}$, Pierre Nora a montré comment cette véritable lame de fond animait nos sociétés occidentales. Audacieuse et stimulante, son idée fut la suivante: mettre en avant le poids du symbolique dans l'univers de la construction historique et, dans la lignée de l'école des Annales, peaufiner les outils pour mettre en scène une « histoire totale ». Ces Lieux de mémoires ont, depuis, fait école. Sans étudier plus avant les questions de réception des œuvres ou encore de celles liées à leurs transmissions, ce travail historiographique a, à partir de la dialectique Mémoire/Histoire, mis en avant de façon magistrale les différentes représentations du passé et les usages distincts de la temporalité. Dans cet univers, le "patrimoine immatériel ${ }^{2}$ » composé de traditions orales, ou encore prenant la forme de spectacles vivants, gagne aujourd'hui lui aussi le devant de la scène ${ }^{3}$.

3 Le monde de la danse n'échappe pas à cette dynamique. Mais comment s'actualise-t-elle, et quels en sont les enjeux lorsque l'on pose la question à l'Histoire, à l'Esthétique ou encore au Droit? Fêtant dernièrement leur centenaire, L'après-midi d'un faune - créé en 1912 - et le Sacre du printemps - scandalisant le Tout-Paris en 1913 - permettent de saisir, au singulier, les aspects universels de ce moment. Ici, des univers techniques différents mettent en scène des intérêts et des visions du monde qui souvent divergent. Au cœur de ces controverses et de ces passions, au nom de l'Histoire ou de la Mémoire, les œuvres et le nom de Vaslav Nijinski gagnent le haut de l'affiche. 


\section{D'une Étoile à l'autre : la mémoire visitée} « conservatoires » et le répertoire, les corps dansants sont souvent perçus, pensés et travaillés comme de véritables archives ouvertes, sur le passé bien sûr, mais aussi sur le futur (bon nombre des «œuvres corps » d'aujourd'hui seront le patrimoine de demain). Déjà, dans le labeur quotidien des studios, chaque danseur se révèle, se compose et «s'organise » en incorporant les multiples idéaux des traditions qui donnent sens à leur univers. Liée à une anatomie politique du détail (réelle ou fantasmée), chaque transmission opère par strates: celles d'un passé sédimenté à jamais ouvert sur le présent. Façonnant les corps, ces visions du monde et de l'art lient ainsi leur destin à la gestion savante d'une bio-esthétique pensée idéale. Aussi, dans cet univers de savoirs et de savoir-faire, exhumer un passé, c'est ressusciter, sous l'emprise d'une culture orale (puissante mémoire des maisons d'opéra), la part d'une histoire de la danse lovée au creux des os et des chairs pour, du même geste, espérer retrouver en soi la vision du monde que jadis elle avait offerte. D'une étoile à l'autre dans le décours du temps, les corps se transmettent ainsi leurs héritages sur les scènes du monde. Dès lors, la postérité se révèle dans la réincarnation quasi mystique d'altérités défuntes. Wilfried Romoli se confie sur ce point: "Cela pose un problème. Comment faire vivre l'œuvre, sans la dénaturer? (...) La difficulté est de respecter "l'esprit de l'œuvre", transmettre ce que la chorégraphe avait envie de raconter, lorsqu'il y a quelque chose à raconter. Il y a tous ces paramètres à gérer... C'est difficile. J'ai acheté l'ouvrage où il y a toutes les photos du Baron Adolphe de Meyer pour voir les détails des mains, en essayant de deviner : « ce que je vois, qu'est-ce au regard de ce que je fais? ». J’ai fait ce travail-là... Puis, j'ai estimé que Charles Jude avait la version musicale la plus logique par rapport à ce que je voyais. (...). En revanche, j'ai choisi d'aller dans la direction de l'interprétation de Rudolf Noureev et pas dans celle de Charles Jude. (...) Donc, je l'ai fait beaucoup plus animal que Jude et je suis allé dans la veine de Rudolf... J'ai pris cette décision, ça a très bien marché d'ailleurs, c'était un de mes très beaux rôles que j'ai dansés (...) [et plus loin] Il s'agit de trouver son soi, de trouver sa danse ${ }^{6}$. Cette démarche n'est bien sûr pas étrangère à ce qui constitue le fondement de l'univers de la danse classique. À l'écoute d'un passé perçu par héritage et par filiation, « l'Étoile » n'a de cesse d'incorporer le corps des Étoiles l'ayant précédées tout en tentant, dans un geste de dépassement, d'affirmer au singulier l'incarnation d'un nouvel idéal. Car ici, l'objet transmis par héritage ne cesse d'être vivifié par le souffle du présent.

6 Filiation dans la filiation, ce travail mémoriel reste pour l'Étoile une conscience de soi, de sa place et de ses responsabilités au cœur même de l'événement. Cependant, si cette éthique corporelle s'assume en tant que telle pour produire de l'excellence corporelle, si 
elle accorde aux incarnations du faune un surplus d'existence, ce travail singulier occulte un pan de l'univers dramaturgique de cette œuvre devenue célèbre. Car si le travail de la mémoire tisse un lien entre le passé et le présent, s'il crée de la filiation par le souvenir, ce dernier n'est pas exempt de distorsions et d'oublis. Qu'on en juge !

7 Archives et reconstitutions en témoignent: le Faune et les nymphes dansent sur un espace plan. Cependant, à l'image de la toile de fond de scène conçue par Léon Bakst, travaillant la profondeur en maniant savamment les masses de clair et d'obscur (et non plus selon les lois de l'optique et de la perspective), le chorégraphe invente, lui aussi, une nouvelle sémiologie. D'autres paramètres créent entre les personnages une véritable « distance ». Romola Nijinsky en a fait état : «Pour le Faune, on choisit sept filles; leur taille avait de l'importance dans ce choix ; elles devaient, en effet, s'égaler à peu près, le ballet était composé sur un seul plan, comme un bas-relief. On donna le rôle de la première nymphe à Nelidova, la plus grande de toutes les danseuses, qui forma ainsi le centre de la frise. Bronia devait jouer la nymphe qui rencontre le faune et s'enfuit prise de panique - rôle petit, mais difficile dont elle fit une présentation très remarquable ${ }^{7}$.» Selon l'épouse du chorégraphe, la taille des nymphes reste proportionnelle à l'importance de leur rôle. Ainsi, en réunissant des danseurs de stature différente, Vaslav Nijinsky met $\mathrm{du}$ sens à son univers plan. Le grand et le petit, devenus à l'occasion l'essentiel et le contingent, se substituent aux catégories du loin et du près. La nymphe principale, bien sûr la plus grande, dépasse le Faune lui-même. L'artiste l'a voulu ainsi.

Occultant en partie un pan de la dramaturgie, le Faune remonté à l'Opéra témoigne plus de l'histoire des $\operatorname{corps}^{8}$, de son éthique, de sa technique et de ses évolutions que de l'histoire de ce spectacle singulier?.

\section{« Éclats » de mémoire : l'interprétation poétique d'une pensée de l'histoire}

Quelques années plus tard cependant, une poignée de danseurs contemporains désavouent avec fermeté l'évidence «muséale » de cette reconstitution traditionnelle. Christophe Wavelet taille sa plume et lance ses traits. Dans la revue Mouvement, il dénonce la façon de procéder en vigueur dans le monde du ballet : «(...) il importe assez peu, en dernier recours, que celui que nous voyons transmettre cette danse 㕆Charles Jude et le Faune繄 se soit inutilement privé de l'appui que lui offrait pourtant l'existence de la partition chorégraphique que Nijinski prit soin d'établir patiemment. De surcroît, que nous le prenions en flagrant délit de fabuler, au nom de la « mémoire » qu'il a conservée de cette danse (c'est-à-dire, en réalité, de l'idée qu'il s'en est faite) n'a non plus rien, en soi, de définitivement disqualifiant. Non, le plus triste, c'est plutôt la médiocrité artistique de cette fiction chorégraphique à prétention historique ${ }^{10} »$. En posant l'Histoire comme question et non comme filiation, le chercheur-danseur place sa critique dans le domaine de l'épistémologie. Déniant une quelconque légitimité historique à ce Faune « remonté » à l'Opéra de Paris, il désavoue d'un même élan les modes de transmission orale et mimétique de l'académisme. Selon lui, cette quête des origines reste aussi vaine qu'illusoire. Pour ce " contemporain », le passé, plus électif qu'impératif, n'est pas un ensoi à retrouver pour le montrer, mais une fiction du monde qu'il convient toujours de questionner selon les règles de l'historiographie. 

quatuor Albrech Knust ${ }^{12}$, représentent bien cette tendance. En 2002, «...D’un faune (Éclats ) », leur proposition chorégraphique, pose d'emblée à L'après-midi d'un faune la question de sa trace et de sa mémoire. Et puisque chacun à sa mesure, Stéphane Mallarmé, Claude Debussy et Vaslav Nijinski avaient, sous un presque même titre ${ }^{13}$, mis en mots, en notes et en pas un pan de la vie de ce faune singulier, ils en analysent la «modernité » en se référant aux «trois poèmes » actualisés en trois genres différents : littéraire, musical et chorégraphique.

11 Deux ans et demi de recherches sur les réceptions successives et distinctes de ces Faunes accompagnent les tâches relatives au déchiffrement de la partition initiale élaborée par Vaslav Nijinski dans les années 1915, et retranscrite en labanotation par Ann Hutchinson Guest et Claudia Jeschke à partir de $1983^{14}$. Exploitant les sources retrouvées, les danseurs cherchent des résonances. Le rideau se lève ${ }^{15}$. Réalisés d'après les maquettes de Léon Bakst, les costumes projettent le parterre dans l'atmosphère de la Belle Époque. Des éclairages «impressionnistes" soulignent le trait. Ici, un tulle pour évoquer la déconstruction de la profondeur de l'espace scénique voulue par Vaslav Nijinski. Là, un Faune-femme pour faire écho à la performance de Bronislava Nijinska dansant, en 1922, le rôle-titre à la demande de Serge de Diaghilev. Des «mots-fragments", issus de Pour un tombeau d'Anatole de Stéphane Mallarmé, s'impriment sur le décor. Parodiant un couple de bourgeois sortant d'une exposition consacrée à l'érotique des vases grecs de la Grèce Antique, Emmanuelle Huynh et Loïc Touzé entament un duo en écho aux accusations de Gaston Calmette, le directeur du Figaro, qualifiant "d'indécent» le "tableau chorégraphique » du danseur russe. Plus loin, un opus de Chopin, pour marquer le souvenir de la dernière apparition de Nijinski improvisant, sur un prélude du maître, une danse à Saint-Moritz (...).

À chaque éclat, une mémoire ; à chaque mémoire, un passé reconsidéré. Dans la mise en tension subtile de ces multiples identités, ces Éclats revisitent, en l'interprétant, la péripétie de ce faune mythique. Et toujours, sous une même appellation, des mots, des notes et des pas. Lors de leurs répétitions, les membres du Quatuor Albrech Knust invitent des amis. Des liens se tissent. Sabine Prokhoris, rencontrée par Christophe Wavelet, lecteur attentif de Michel Foucault, lors de leur aventure commune à la direction de la revue Vacarme, partage parfois, avec d'autres, ces instants où la création se fait. Psychanalyste et agrégée de philosophie, l'ancienne secrétaire du centre Michel Foucault participe aux conversations et autres discussions. Sans être totalitaire pour autant, inéluctablement, la pensée de Michel Foucault nimbe l'atmosphère de ces réunions de travail. Au programme : la danse bien sûr, mais aussi une épistémologie de l'Histoire organisée à partir d'un réel sans cesse reconstruit. Non plus une histoire du devenir, mais une histoire visant à la récupération du passé dans l'ici et le maintenant. Car ce type d'histoire n'instaure pas le relativisme de l'objet esthétique, simplement, il prend conscience du relativisme de l'Histoire elle-même ${ }^{16}$. Aussi, danseurs-chercheurs ne prétendent pas exhumer une histoire enfouie pour la montrer à nouveau. Instaurant des rapports d'identités et de différences, ils s'approprient le révolu de façon singulière pour faire apparaitre une série indéfinie de sens historiques. Dans la gestion savante des mouvements, des images et des sons, ces artistes articulent, sur un mode inédit, des manières de dire et de faire à jamais perdues. Engagé dans cette forme d'épistémologie pratique, le vrai gain de l'historiographie présume, selon eux, la perte inéluctable de ce qui a été, car, à ce moment, envisager le passé suppose de l'accommoder pour faire, sous 
une forme poétique et singulière - ils n'oublient pas d'être artistes ! -, de cette mémoire perdue un événement du présent. Pourquoi choisir ces traces et pourquoi retenir ces thématiques? Peu importe! Au nom d'une critique dynamisant du même geste les imaginaires créateurs, il s'agit avant tout de produire un acte poétique ${ }^{17}$.

13 Peaufinant les écarts, ces multiples visions de faunes instaurent des rapports d'identité/ différence entre des chefs-d'œuvre passés et leurs mémoires visitées. En ces appropriations plurielles, l'art chorégraphique invente et déplace. Au creux des corps, des images et des sons, il articule des dires et des faires sur ce que l'obscur du passé a tu à jamais. Ce regard critique opère d'autant mieux, qu'à ce moment, l'heure est à l'échange. Chacun apporte sa marque, ses singularités, ses amitiés.

\section{(Re)présenter le patrimoine : le chorégraphe et I'historien}

2013 : l'année centenaire du Sacre du printemps, chorégraphié par Vaslav Nijinski sur la musique d'Igor Stravinski et des costumes et décor de Nicolas Rœrich, donne au mois de mai une teneur particulière. Pour l'occasion Tamara Nijinski, la fille cadette de Tamara et de Vaslav Nijinski, prend sa plume : «Le 29 mai 1913, au Théâtre des Champs-Elysées, mon père, Vaslav Nijinski, Igor Stravinski et Nicolas Roerich, créaient Le Sacre du printemps, une pièce révolutionnaire tant du point de vue musical, que chorégraphique et esthétique. On sait le scandale que provoqua ce ballet d'avant-garde, dont la modernité ne cesse de surprendre encore aujourd'hui. (...) Il fallut ensuite attendre 1987, pour que Le Sacre du printemps de Vaslav Nijinski fasse à nouveau parler de lui. On doit cette résurrection à une collaboration entre Robert Joffrey, alors directeur du Joffrey Ballet de Chicago, la chorégraphe et historienne de la danse américaine Millicent Hodson et l'historien de l'art anglais, Kenneth Archer. Hodson et Archer entreprirent des recherches qui leur permirent de "reconstituer" le Sacre dans sa version "originale". C'est du moins ainsi que furent annoncées les représentations de ladite "reconstitution"18. ${ }^{18}$ Commémorée dans le lieu même où l'œuvre fit scandale, cette reconstitution proposée mérite un détour.

Nijinski n'ayant pas noté sa chorégraphie, le travail de Millicent Hodson et de Kenneth Archer s'instruit de différentes sources : dessins, photographies, témoignages, partition annotée de Stravinski, une autre annotée par Marie Rambert, assistante de Nijinski à la création du Sacre en 1913, et indiquant certains mouvements, une lettre de Bronislava Nijinska se rapportant à la danse finale de "l'Élue", etc. Seize années, dont sept consacrées à la recherche d'archives et à la récolte de fonds, serviront l'entreprise. À terme, et conformément au droit anglo-saxon, les deux chercheurs déposeront le copyright de cette reconstitution et produiront bon nombre d'articles sur la question. Voilà l'essentiel de l'affaire. Tout d'abord, mettant en évidence certains usages de la modernité, Kenneth Archer ${ }^{19}$ souligne comment le peintre Nicolas Rœrich, archéologue et spécialiste d'histoire de l'art, précisa la thématique de ce Sacre. Ses connaissances donnent sens à une œuvre où totems, ancêtres, rituel sacrificiel etc., ordonnent la vie d'une peuplade primitive au sortir de l'hiver. À la lueur des sources exhumées, Millicent Hodson réinvente les gestes créés par Vaslav Nijinsky loin de tout académisme. À la fin de l'acte deux, la danse du sacrifice témoigne bien de la teneur de cette œuvre iconoclaste, car 
l'artiste avait commencé son étude par ce final en explorant une technique de corps qui donnera son souffle et sa logique à toute la pièce.

En fond de scène, la toile conçue par Nicolas Rœrich dévoile un site singulier : celui du paganisme slave et de ses symboles ${ }^{20}$. Une colline dressée, un cercle de pierres. En une fusion allégorique des éléments de l'univers, la terre côtoie le ciel. En ce point précis, le cosmo-tellurisme semble le plus vif. Sanctuaire immuable, le site s'impose comme " centre» d'une théogonie païenne ${ }^{21}$. Sur le plateau, en écho à ce symbole, un cercle. Située en son centre, "l'Élue». L'image s'impose, obsédante; longtemps le temps s'égraine. Après plus de quatre minutes, la danseuse s'anime, s'arrachant de la terre dans la violence d'un saut. Sans cesse, elle cherche à s'éloigner de ce point qui toujours l'attire. Elle va mourir ; et cela se voit. Sa danse meurtrit sa chair. Soudain un saut, puis un autre. À chaque fois, l'artiste retombe parfois sur les talons, puis repart dans une agitation frénétique. Tordue, agitée, convulsée, elle danse à en perdre force. Contorsions, gestes «d'ataxique ${ }^{22}$, spasmes incessants, son corps porte les traces du déchirement, de la souffrance aussi : sur scène, il exhibe ce qui, d'habitude, se terre. Cette danse de l'excès et de la démesure travaille le pathos. Effort sublime, mais vain, car les forces cosmiques la dominent. Lorsqu'elle s'ouvre vers le ciel ou se ferme vers la terre, elle oriente sa poitrine vers le haut ou vers le bas tout en fléchissant les membres inférieurs. L'ouverture ou les fermetures du torse tirent leurs puissances de ces étirements qui - techniquement - sont autant d'enracinements. L'œil et le buste s'orientent vers les espaces suggérés. La mise sur orbite du regard prolonge l'intention; la poitrine « voit» le ciel (ou encore la terre). Sans être sacrifié pour autant, le visage accompagne cette organisation corporelle qui met à mal l'hégémonie des seules rectitudes et des verticalités de la danse classique. Avec son Faune, déjà, Nijinski avait de la sorte déjoué les usages : sur scène, lorsque son fils de Pan regarde un objet - du raisin -, ou un être - une nymphe -, il le fait surtout avec ses membres supérieurs pointés vers la direction (si les deux bras sont mobilisés, ils forment alors deux parallèles). D'un même geste, l'artiste déplace les foyers d'intention et d'attention. Avec lui, l'œil n'a plus le primat du regard ${ }^{23}$ : ici la poitrine, là les bras. Pour Tamara Nijinski, cette reconstitution est tout à fait convaincante.

Convaincue aussi, Dominique Brun propose cependant de mettre en scène une nouvelle lecture sur la base de l'hypothèse suivante : «Évidemment, je bénéficie de tout le travail fait par Hodson et Archer, précise la chorégraphe, qui a obtenu l'aide à la recherche et au patrimoine en danse. Je leur suis reconnaissante de l'énorme défrichage des archives qu'ils ont effectué, mais la découverte de nouveaux documents me permet aujourd'hui de proposer une autre reconstitution du Sacre.» Lesquels? La partition autographe de L'Après-midi d'un faune, écrite en 1915 par Nijinski lui-même: "Cette partition autographe, et c'est la seule, témoigne de façon unique de l'écriture de Nijinski. Elle raconte comment ça bouge chez Nijinski, dans quelle énergie... Et c'est le seul document qui donne ces indications. Lorsqu'on reconstitue aujourd'hui une pièce "à la manière de", il faut repérer la manière du chorégraphe dans ses œuvres précédentes ${ }^{24}$. " « À la manière de » : pour séduisante qu'elle soit, l'hypothèse mérite une dispute ! En effet, si Nijinski est moderne, s'il œuvre dans cet univers vantant les mérites du «nouveau» et de "l'original ", chacune de ses pièces n'est-elle pas à elle seule sa propre référence ${ }^{25}$ ? Par ailleurs, un regard porté sur le processus créateur montre de grandes différences entre le Faune et le Sacre. Dans ses mémoires, la sœur de Nijinski a relaté ses impressions lors de la genèse du Faune: «Je suis comme un morceau d'argile qu'il modèle, me faisant prendre peu à peu les poses qu'il cherche ${ }^{26} »$. Sensations renforcées par le récit des interprètes : 
«Nous avons l'impression d'être sculptées dans de la pierre ${ }^{27}$.» Pour créer, Nijinski adjoint à la vue, le toucher. Dans cet éprouvé, il saisit et organise son œuvre. Avec au bout des doigts et du regard, l'émergence d'un monde. Pour le Sacre il en est tout autrement. Selon Richard Buckle, lorsque Vaslav Nijinski fait travailler le rôle de l'élue à Maria Pilz, il montre les mouvements à l'interprète : «Le poing serré sur le front, il se jetait en l'air dans un paroxysme de crainte et de souffrance. Ses mouvements avaient beau être stylisés et contrôlés, ils n'en étaient pas moins chargés de puissance tragique. Quand Pilz dansa le rôle en public elle "garda l'essentiel" de la démonstration de Nijinski et fit une profonde impression, mais Rambert n'y vit qu'un "pâle chromo" de ce qu'elle avait vue cet après-midi ${ }^{28} »$. D'un côté, une recherche du mouvement où la sensation se lie à l'aspect plastique et visuel des corps, de l'autre, une étude où la mimesis guide les pas de la danseuse devant incorporer des mouvements où prime l'énergie.

Cependant, à chaque lieu ses attentes, ses usages, ses acceptations: à l'Histoire et l'Esthétique, l'Histoire et l'Esthétique, à la danse, la danse. L'historien « refigure » le passé à partir de traces. Son univers admet que la mémoire des œuvres ne peut être envisagée comme la reproduction de ce qui est réellement arrivé ; pour lui, les réalités passées restent à jamais hors de portée. Ce qui a été ne peut être que postulé, refiguré, thématisé et interprété. Travailler sur des traces, c'est accepter qu'il existe des vides, des zones d'ombre, bref du discontinu. Pour le danseur/chercheur devant «reconstituer» une œuvre, il en est tout autrement. Les vides doivent être comblés, les zones d'ombre levées. Car, pour le sens commun, la mémoire d'une œuvre, sa commémoration ou encore sa patrimonialisation exige du continu. Ici à dessein, les hypothèses historiennes peuvent servir de liant pour combler le néant.

\section{Propriété intellectuelle : à qui appartient le Sacre?}

Voici à présent comment la fille de Nijinski poursuit sa missive :

Depuis maintenant plus de vingt-cinq ans, Le Sacre du printemps de Vaslav Nijinski, ou "d'après Vaslav Nijinski", "reconstitué par Millicent Hodson et Kenneth Archer", est donné à travers le monde, il est inscrit au répertoire d'une dizaine des plus prestigieuses compagnies de danse, notamment le Théâtre Mariinski de SaintPétersbourg qui se produira au Théâtre des Champs-Élysées. Depuis maintenant plus de vingt-cinq ans, les héritiers de Vaslav Nijinski, aujourd'hui mon neveu Vaslav Markevitch et moi-même, ne perçoivent aucun droit d'auteur sur cette œuvre, dont Hodson et Archer se prétendent propriétaires au motif que Nijinski n'avait pas noté sa chorégraphie et que leur "reconstitution" est leur seule propriété. Depuis maintenant plus de vingt-cinq ans, le public assiste à des représentations dont la publicité est entièrement faite sur le nom de Vaslav Nijinski au détriment des droits de ses héritiers. (...)

Cette année, grâce à la législation française sur le droit d'auteur, et au Théâtre des Champs-Élysées qui accueille le Sacre, ainsi qu'à la SACD (Société des Auteurs et Compositeurs Dramatiques) j'assisterai pour la première fois à une représentation du Sacre du printemps avec notre autorisation, dans sa chorégraphie dite originale. Ce sera aussi la première fois que la Succession Nijinski percevra des droits d'auteur sur cette pièce de Nijinski, "reconstituée" par Hodson et Archer. (...) J'ajoute que je serai à Paris à l'initiative d'un collectif de chorégraphes contemporains français qui se sont cotisés pour nous permettre, à ma fille et à moi-même, de faire ce voyage et d'assister à la célébration du centenaire du Sacre. Je les remercie avec chaleur ${ }^{29}$.

En aval des fêtes commémoratives se pose la question de la protection des objets de l'art, et plus précisément de celles des œuvres chorégraphiques. En droit, celle-ci passe avant 
tout par la propriété intellectuelle. Selon Jean-Luc Piotraut: «Sera, à ce titre, principalement visée une protection par le droit d'auteur, lequel désigne l'ensemble des prérogatives dont jouissent les créateurs sur leurs œuvres littéraires et artistiques ${ }^{30}$. » À l'heure de la mondialisation, au moment où l'économie de marché dicte ses exigences, la question des droits touche et organise les modes d'existence du monde de l'art. À plusieurs égards, le centenaire du Sacre en révèle les enjeux. Plusieurs "parties » participent à cette commémoration : le Théâtre Mariinski ${ }^{31}$ de Saint-Pétersbourg produit la reconstitution faite par Millicent Hodson et Kenneth Archer, le Théâtre des ChampsÉlysées l'organise. Comme tel, le théâtre de l'avenue Montaigne doit s'assurer que le producteur possède bien les droits. C'est ici que l'affaire se complique puisque d'une durée de « 70 ans post mortem», la question des droits reste pour la famille un enjeu artistique, mais aussi commercial. Qu'on en juge !

21 Alerté par la Société des Auteurs et Compositeurs (SACD) des exigences de la famille de Nijinski, le Théâtre des Champs-Élysées s'est retourné vers le Mariinski qui, surpris, se croyait en règle en acquittant des droits à Kenneth Archer et Millicent Hodson ${ }^{32}$. En effet, en matière de Droit, les lois divergent d'un pays à l'autre : d'un côté le droit anglo-saxon exigeant le dépôt d'un copyright, chose faite, on l'a vu, par Kenneth Archer et Millicent Hodson pour leur reconstitution, de l'autre, le droit français n'imposant pas une telle démarche : «En droit français ${ }^{33}$, la protection du droit d'auteur porte sur ce que le Code de la propriété intellectuelle (CPI) dénomme les « œuvres de l'esprit », notion assimilée par la jurisprudence ${ }^{34}$ à toute création de forme, à l'exclusion des seuls concepts ou idées qui ne donnent dès lors pas prise au droit d'auteur ${ }^{35}$.» Dans cet esprit, un bulletin de déclaration du 9 juin 1913, déposé à la SACD à Paris, avait cependant réparti les droits : deux tiers pour Igor Stravinsky, un tiers pour Nicolas Roerich et Vaslav Nijinski ${ }^{36}$. Le Sacre joué à nouveau à Paris, des droits furent, pour la première fois depuis la première reconstitution de l'œuvre en 1987, attribués aux descendants de Nijinski. Le fait est d'importance, car en la matière, la jurisprudence dicte le droit. Aussi, très vite le sujet déborde le cadre étroit de cette commémoration. Chargée du dossier à la SACD, Corinne Honvault ${ }^{37}$ lance sa mise en garde. Selon elle, les chorégraphes français, dont le statut d'auteurs en France est récent (1991), doivent se rendre sensible à l'événement : «Pour la génération apparue dans les années 1980, la question de la reprise de leurs pièces par des interprètes les ayant dansées peut entraîner des problèmes de prérogatives. Cette affaire choquante devrait les sensibiliser à la protection de leurs œuvres après leur disparition et à la transmission du patrimoine chorégraphique dans le respect de la chaîne des droits ${ }^{38}$.» Certains artistes avaient anticipé - ou contribué à - cet appel. Sous l'égide de Daniel Larrieu, danseur et chorégraphe, mais aussi administrateur danse à la SACD depuis juin 2012, un collectif de chorégraphes se cotise pour participer au voyage de Tamara et sa fille de Phoenix à Paris. Dans ce contexte, la solidarité envers la fille du chorégraphe se charge d'une dimension politique. Car, à «l'ère des commémorations ", la gloire de Nijinski, comme celle de ses pièces, fait évoluer les regards portés sur le monde chorégraphique, sur son excellence et sur sa législation. Dans un monde où notoriété et succès légitiment souvent des décisions de justice fondées de manière prétorienne ${ }^{39}$, le moment profite, à dessein, de l'aura d'une icône et de son œuvre.

On l'aura compris : si ces questions liées à la mémoire et à l'héritage animent les scènes de l'Histoire et de l'Esthétique, elles interpellent bien évidemment le Droit. Nom et notoriété deviennent alors des facteurs décisifs pour faire évoluer un monde où la jurisprudence a force de loi. 
23 Au moment où l'ingénierie culturelle perçoit en cette idéalisation du passé une valeur marchande, l'heure est à la restauration et à la valorisation d'œuvres devenues « chefsd'œuvre " pour la postérité. Sorte de « grands récits » (dont la plupart ont disparu ${ }^{40}$ ), ces derniers, s'immisçant au cœur de la mémoire collective, semblent sceller l'identité d'un groupe. Du même geste, ils renforcent le mythe d'une société unifiée.

Cependant, au nom de ces objets du passé, des éthiques et des esthétiques s'affrontent sur scène. D'un même élan, elles révèlent combien nos sociétés relèvent d'une pluralité d'identités. D'une lecture à l'autre, d'une œuvre à l'autre, classiques et contemporains imposent des légitimités qui parfois s'affrontent sans toujours se rencontrer. D’un côté, une approche historienne trouvant ses hypothèses dans l'épistémè du moment : le passé se regarde alors par le filtre du présent, les archives exhumées puis déchiffrées imposent des discontinuités dans lesquelles se glissent des visions poétiques d'un passé de la danse. De l'autre une logique de la mémoire et de l'héritage « œuvrant » pour la totalité, le continu et le causal. Ici et là cependant, ces histoires ou ces mémoires se lient à une histoire plus souterraine : celle de l'affirmation de l'être comme conscience de soi et sujet de lui-même.

\section{NOTES}

1. Les Lieux de Mémoire parus chez Gallimard entre 1984 et 1992.

2. La notion apparaît au début des années 1990. En 2003, la Convention pour la sauvegarde du patrimoine culturelle et immatériel sera adoptée par l'UNESCO.

3. Voir Pierre Nora, Présent, nation, mémoire, Paris, Gallimard, 2011, p. 99.

4. Ici, nous reprenons, en partie, un passage traité dans Roland Huesca, «Héritage et postérité, l'après-midi d'un faune de Nijinski ", Roland Huesca, Olivier Goetz, Chefs-d'œuvre!/?, Paris, Nouvelle éditions Jean-Michel Place, 2013, p. 145-151.

5. Ce n'est donc pas une captation in situ, pour preuve : il n'y pas le décor.

6. Entretien avec Wilfried Romoli, 15 mai 2013.

7. Romola Nijinsky, Vaslav Nijinsky, Paris, Denoël et Steele, 1934, p 157.

8. On peut faire la même remarque concernant le travail opéré en 2006 par Dominique Brun. À partir de la partition chorégraphique laissée par Nijinski en 1915, l'artiste a focalisé son attention et son intérêt sur l'histoire de ce corps, de ces corps, refiguré à partir de ces retranscriptions, mais aussi des ports de tête singuliers imposés par la lourde perruque portée par Nijinski. Chemin faisant, elle s'est émancipée du décor et des costumes conçus par Léon Bakst (pas de toile de fond - mais sur ce point, les budgets font parfois défaut! - et Faune dépourvu ici des protubérances phalliques, qui, sur le costume, octroyaient au chèvre-pied un surplus de vigueur). Quasiment dépourvu de ces attributs singuliers, et sans le décor de référence, le faune de Dominique Brun construit avec talent une autre histoire : celle où prime, par la notation, la recherche des usages de l'autre en soi. Mais de quoi, et de qui, ce travail est-il le nom ?

9. Totalement singulière dans le monde de la danse, la démarche de Merce Cunningham est significative. Son "Legacy plan", testament laissé par lui à sa mort, est évocateur. Chaque « danse capsule », véritables «valises pédagogiques numériques », contiennent tous les éléments 
concernant un spectacle: captation vidéo, prises de notes, dessins, musiques, indications de décors, costumes, lumières, etc...

10. Christophe Wavelet, « Nijinski, encore », Mouvement, janvier/mars 2001, p. 109-111.

11. Dans le cadre d'un examen sur la cinétographie Laban au conservatoire de la Villette, sont recréées, au début des années 90, des pièces de Doris Humphrey Two ecstatic themes, (1931), et Invention (1949)

12. Le quatuor reprend le nom du danseur, chorégraphe et pédagogue allemand qui consacra une partie de son activité à développer le système de notation du mouvement dont les principes furent élaborés par Rudolf Laban.

13. L'œuvre de Claude Debussy est intitulée Prélude à l'après-midi d'un faune.

14. Claudia Jeschke et Ann Hutchinson Guest, "Waslaw Nijinski chorégraphe du faune », in Françoise Stanciu-Reiss Jean-Michel Pourvoyeur, Écrits sur Nijinski, Paris, Chiron, 1992, p. 79-88.

15. Pour décrire ces bribes de scènes, les multiples précisions et détails donnés par Christophe Wavelet furent précieux. Nous l'en remercions.

16. Nous avons repris ici, un thème développé plus avant dans Roland Huesca, « Michel Foucault et les chorégraphes français ", Strasbourg, le portiQue, 13-14, 2004, p. 237-249.

17. Si au début du XXe siècle, les joutes entre les classiques et les modernes se faisaient au nom de la technique, les voici désormais portées également sur le front de l'épistémologie.

18. « Lettre de Tamara Nijinski », Le Monde, 29 mai 2013. Article consultable à l'adresse suivante <http://www.lemonde.fr/culture/article/2013/05/27/une-lettre-de-tamaranijinski_3418210_3246.html>.

19. Archer K., " Nicolas Roerich », in Souriau É. (et al.). Le Sacre du Printemps de Nijinsky, Paris, Cicero et Théâtre des Champs-Élysées, 1990, p. 75-99.

20. Kenneth Archer, qui a reconstitué les décors et les costumes de l'œuvre, a bien montré comment le peintre Nicolas Rœrich, très inspiré par les rites slaves, proposait souvent dans ses toiles des paysages de ce type avec des pierres disposées sur le haut de collines. Voir Archer K., loc. cit., p. 75-95.

21. Éliade M., dans Le Mythe de l'Éternel Retour, 1947, trad. Gouillard J. et Soucasse J., Paris, Gallimard, 1969, p. 24 et suiv., a magistralement développé ce « Symbolisme du centre. »

22. L'expression est d'époque. Elle renvoie aux problèmes de coordination. Le terme est employé par exemple par Souday P., « Théâtre des Champs-Élysées », in L'Éclair, 31 mai 1913. et par Lalo P., « La Musique », in Le Temps, 5 août 1913.

23. Jacques Rivière en fait échos. Selon lui, jusque-là, pour communiquer une émotion, le danseur mobilisait des mimiques : "sourcils froncés ou bien sourire ", dans "Le Sacre du Printemps", Nouvelle Revue Française, 7 novembre 1913, p. 706-730.

24. Citée par Rosita Boisseau, « Dans les pas de Nijinski », Le Monde, 25 mai 1913.

25. C'est ce que nous avons tenté de montrer dans Triomphes et scandales la belle époque des ballets, Paris, Hermann, 2001.

26. Dans B. Nijinska, Mémoires 1891-1914, trad. G. Mannoni, 1981, Paris, Ramsey, p 280.

27. Ibid., $p 379$.

28. Richard Buckle, Diaghilev, 1979, trad. Tony Mayer, Paris, Jean-Claude Lattès, 1980, p. 293.

29. «Lettre de Tamara Nijinski », Le Monde, 29 mai 2013, Op. cit.

30. Jean-Luc Piotraut, «La protection des chefs-d'œuvre par le droit d'auteur », Roland Huesca, Olivier Goetz dir., Chefs-d'oevure!/?, Paris, Les nouvelles éditions Jean-Michel Place, 2013, p. 125-136. Ici, la plupart des informations sur le droit sont tirées de cet article

31. On le nomme aussi le Kirov.

32. Ariane Bavelier, « Foire d'empoigne autour du Sacre du printemps », Le Figaro, 29 mai 2013.

33. Conformément à l'article L. 112-1 Du Code la propriété intellectuelle.

34. C'est-à-dire l'ensemble des décisions judiciaires rendues en la matière. 
35. Jean-Luc Piotraut, "La protection des chefs-d'œuvre par le droit d'auteur ", in Roland Huesca, Olivier Goetz dir., Chefs-d'œuvre !/ ?, op. cit., p. 125-136.

36. Ariane Bavelier, « Foire d'empoigne autour du Sacre du printemps », Le Figaro, 29 mai 2013.

37. De la SACD.

38. Rosita Boisseau, «Les faux-pas de la succession de Nijinski», Le Monde, 29 mai 2013. Article consultable à l'adresse : http://www.lemonde.fr/culture/article/2013/05/27/les-faux-pas-de-lasuccession-nijinski_3418115_3246.html

39. Voir Jean-Luc Piotraut, loc. cit., p. 125-136.

40. Voir Jean-François Lyotard, La condition postmoderne, Paris, Minuit, 1979.

\section{RÉSUMÉS}

Désirs de mémoire, de reconstitution ou encore de commémoration, depuis la fin du $\mathrm{XX}^{\mathrm{e}}$ siècle, la société française s'enivre de son passé. En une série d'ouvrages désormais célèbres, Pierre Nora a montré comment cette véritable lame de fond animait nos sociétés occidentales. Le monde de la danse n'échappe pas à cette dynamique. Rien n'est simple pour autant ! Prenant pour objet les œuvres de Nijinski, « classiques » et « contemporains » mettent en scène des intérêts, des visions du monde et des légitimités qui souvent divergent. D'un côté, une approche poétique d'une intelligence de l'histoire construisant ses hypothèses dans l'épistémè du moment. De l'autre, une logique de la mémoire et de l'héritage « œuvrant » pour la totalité, le continu et le causal. Ici et là cependant, l'éthique singulière de ces corps dansant se lie à une histoire plus souterraine : celle de l'affirmation de l'être comme conscience de soi et sujet de lui-même.

\section{INDEX}

Mots-clés : droits d'auteur, Nijinski (Vaslav), Sacre du printemps (Le), reprise, scandale

\section{AUTEUR}

\section{ROLAND HUESCA}

Professeur d'Esthétique et directeur du département « Arts » de l'Université de Lorraine, Roland Huesca travaille en priorité sur les questions relatives à l'histoire et à l'esthétique du spectacle vivant. Il a publié en 2012 Danse, Art et modernité : au mépris des usages (Paris, PUF, coll. Lignes d'art,2012), et a écrit l'ouvrage La danse des orifices : étude sur la nudité, à paraître fin 2014 (Paris, Les nouvelles éditions Jean-Michel Place). 